\title{
Point discharge current measurements beneath dust devils
}

\section{Article}

\section{Accepted Version}

Creative Commons: Attribution-Noncommercial-No Derivative Works 4.0

Lorenz, R. D., Neakrase, L. D. V., Anderson, J. P., Harrison, R. G. and Nicoll, K. A. (2016) Point discharge current measurements beneath dust devils. Journal of Atmospheric and Solar-Terrestrial Physics, 150-151. pp. 55-60. ISSN 13646826 doi: https://doi.org/10.1016/j.jastp.2016.10.017 Available at https://centaur.reading.ac.uk/67963/

It is advisable to refer to the publisher's version if you intend to cite from the work. See Guidance on citing.

To link to this article DOI: http://dx.doi.org/10.1016/j.jastp.2016.10.017

Publisher: Elsevier

All outputs in CentAUR are protected by Intellectual Property Rights law, including copyright law. Copyright and IPR is retained by the creators or other copyright holders. Terms and conditions for use of this material are defined in the End User Agreement.

\section{www.reading.ac.uk/centaur}

\section{CentAUR}

Central Archive at the University of Reading 
Reading's research outputs online 


\title{
Point Discharge Current Measurements beneath
}

\section{Dust Devils}

\author{
Ralph D. Lorenz*
}

Johns Hopkins University Applied Physics Laboratory, 11100 Johns Hopkins Road, Laurel, MD 20723, USA.

Lynn D. V. Neakrase

Department of Astronomy, New Mexico State University, Las Cruces, NM, USA

John P. Anderson

Jornada Experimental Range Headquarters, New Mexico State University, Las Cruces, NM, USA

R. Giles Harrison, Keri A. Nicoll

Department of Meteorology, University of Reading, UK

*Corresponding Author. Tel.+1 4437782903 Fax. +1 4437788939 Email: Ralph.lorenz@jhuapl.edu

Submitted to Journal of Atmospheric and Solar-Terrestrial Physics, August 5, 2016

Assigned MS ATP-S-16-00311. Revised Version, October 26, 2016 
18 Abstract

19 We document for the first time observations of point discharge currents under dust devils using a novel compact sensor deployed in summer 2016 at the USDA-ARS Jornada Experimental Range in New Mexico, USA. A consistent signature is noted in about a dozen events seen over 40 days, with a positive current ramping up towards closest approach, switching to a decaying negative current as the devil recedes. The currents, induced on a small wire about $10 \mathrm{~cm}$ above the ground, correlate with dust devil intensity (pressure drop) and dust loading, and reached several hundred picoAmps. 
29 ${ }_{55}$
1. Introduction

Dust devils are an important agent of dust-raising on Earth and Mars (Balme and Greeley, 2006). On Mars, they are among the most prominent meteorological features, whereas on Earth they are mostly a curiosity but sometimes cause damage or, very occasionally, fatalities (Lorenz et al., 2016). Triboelectric processes typically lead to charging of the lofted dust (Harrison et al., 2016), and even from the earliest days of systematic studies of dust devils, their electrical properties have been of interest. A notable example in the early work is that of Colonel Baddeley of the British Army, who in the 1860s, carried a gold-leaf electroscope into dust devils in India (e.g. Baddeley, 1860; Lorenz et al., 2016).

Interest in the electrical properties of dust devils at Mars has been stimulated by the notion that tribochemistry and/or electrical discharges may influence atmospheric chemistry, via the production of oxidants which may play a role in the destruction of organics and/or methane (e.g. Atreya et al., 2006; Delory et al., 2006; Kok and Renno, 2009). In fact early investigators such as Baddeley and others also studied the presence of ozone in terrestrial dust devils (Lorenz et al., 2016), hence understanding the electrical and chemical aspects of dust devils can be appreciated as of enduring importance. The recently-launched European Space Agency (ESA) ExoMars Schiaparrelli lander brings new interest in dust devil electrification, in that its DREAMS meteorology package (Dust Characterisation, Risk Assessment, and Environment Analyser on the Martian Surface, Esposito et al., 2014) includes an electric field sensor, with the prospect of providing Mars data to compare with conceptual (e.g. Farrell et al., 2003) and numerical (e.g. Barth et al., 2016) models. so (1) 5 54 
2. Site and Instrumentation

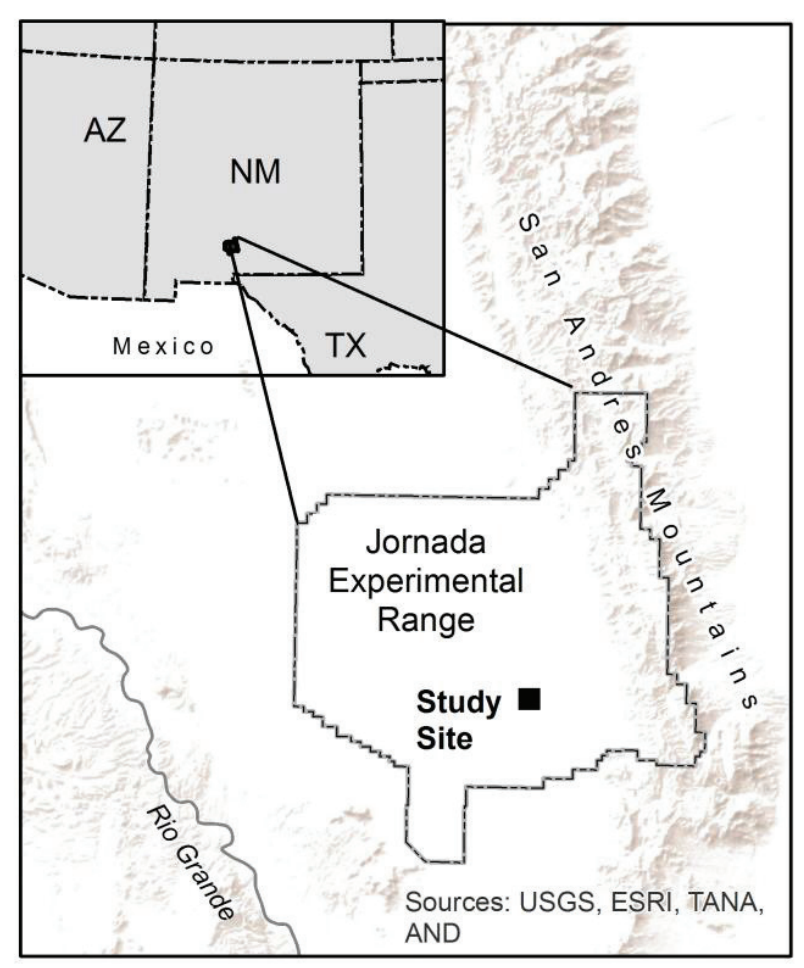

66

Figure 1. Study Site Location Observations were made between 4 May and 14 June 2016 at the Jornada Experimental Range (783 $\mathrm{km}^{2}$ ), $37 \mathrm{~km}$ north of Las Cruces, NM. This US Department of Agriculture facility on the Jornada del Muerto Plain lies between the Rio Grande floodplain (elevation 1,186 m) on the west and the crest of the San Andres Mountains (2,833 m) on the east. Just beyond the San Andres Mountains are the dunes of White Sands National Monument, and the nearby missile range, which was the site of a previous visual dust devil survey (Snow and McLelland, 1990). In fact, the Jornada del Muerto itself was the site of measurements of dust devil electric fields made a half century ago (Crozier, 1970).

68 The climate of Jornada is characteristic of the northern region of the Chihuahuan Desert with abundant sunshine, low relative humidity, wide diurnal temperature ranges (average maxima are $36^{\circ} \mathrm{C}$ in June), 
70

71

72

73

74 and variable precipitation both temporally and spatially. Potential evaporation is approximately 10 times the average precipitation, which is $241 \mathrm{~mm} \mathrm{yr}^{-1}$ and occurs as localized thunderstorms during July, August, and September. The site $\left(106.69016^{\circ} \mathrm{W}, 32.58752^{\circ} \mathrm{N}\right)$ has variable cover of grasses and low scrub (figure 2).

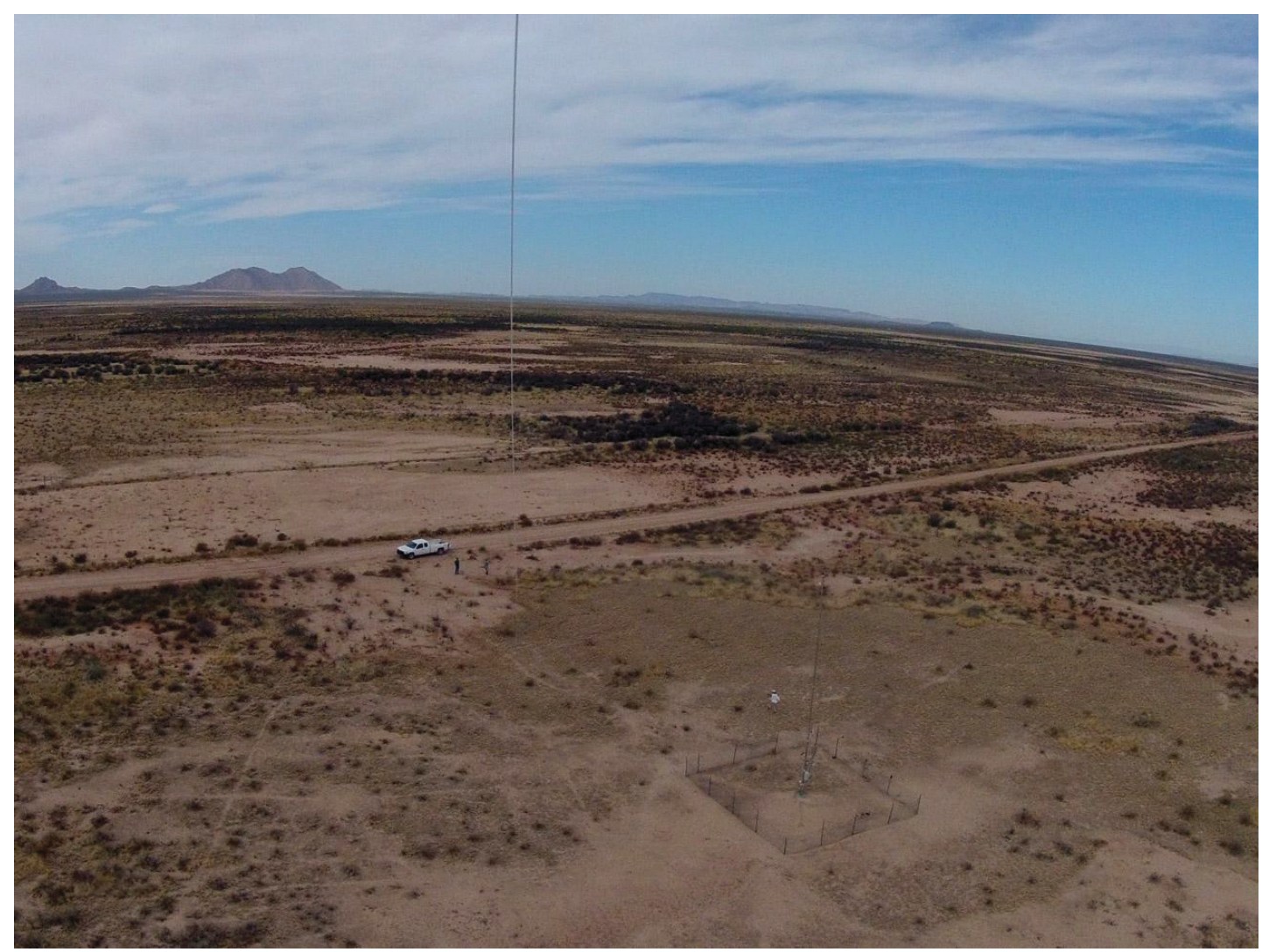

Figure 2. Aerial view of the field site, taken with a GOPRO digital camera lofted on a parafoil kite (the kite string is visible just left of center). The area is flat, with partial cover of scrub land bushes. The loggers were deployed just to the lower right of the square fence that protects an unrelated meteorological installation. A vehicle and research personnel are visible for scale.

Following previous successful deployments (Lorenz et al., 2015; Lorenz, 2016) at Jornada, we use Gulf Coast Data Concepts B1100 pressure loggers, which monitor a precision Bosch BMP085 pressure sensor (recorded with a resolution of $1 \mathrm{~Pa}$, or $0.01 \mathrm{mbar}$ ) with a microcontroller that logs the pressure data and housekeeping temperature as ASCII files on a 2 GB microSD flash memory card. The whole unit operates 
as, and its form factor resembles, a large USB memory stick, facilitating data transfer to a PC. As described in Lorenz (2012), for this application the nominal single AA battery is replaced by a pair of alkaline D-cells (figure 3), allowing unattended multi-month operation at sample rates of $2 \mathrm{~Hz}$ or more. The sensor and battery are installed in a plastic case, drilled to allow pressure equalization. An augmentation to the standard B1100 that was made available to us by the manufacturer (see e.g. Lorenz and Jackson, 2015) is the option to record an additional analog voltage (in the range 0 to $4.19 \mathrm{~V}$ ) with 12 bit resolution at an interval of $1 \mathrm{~s}$.

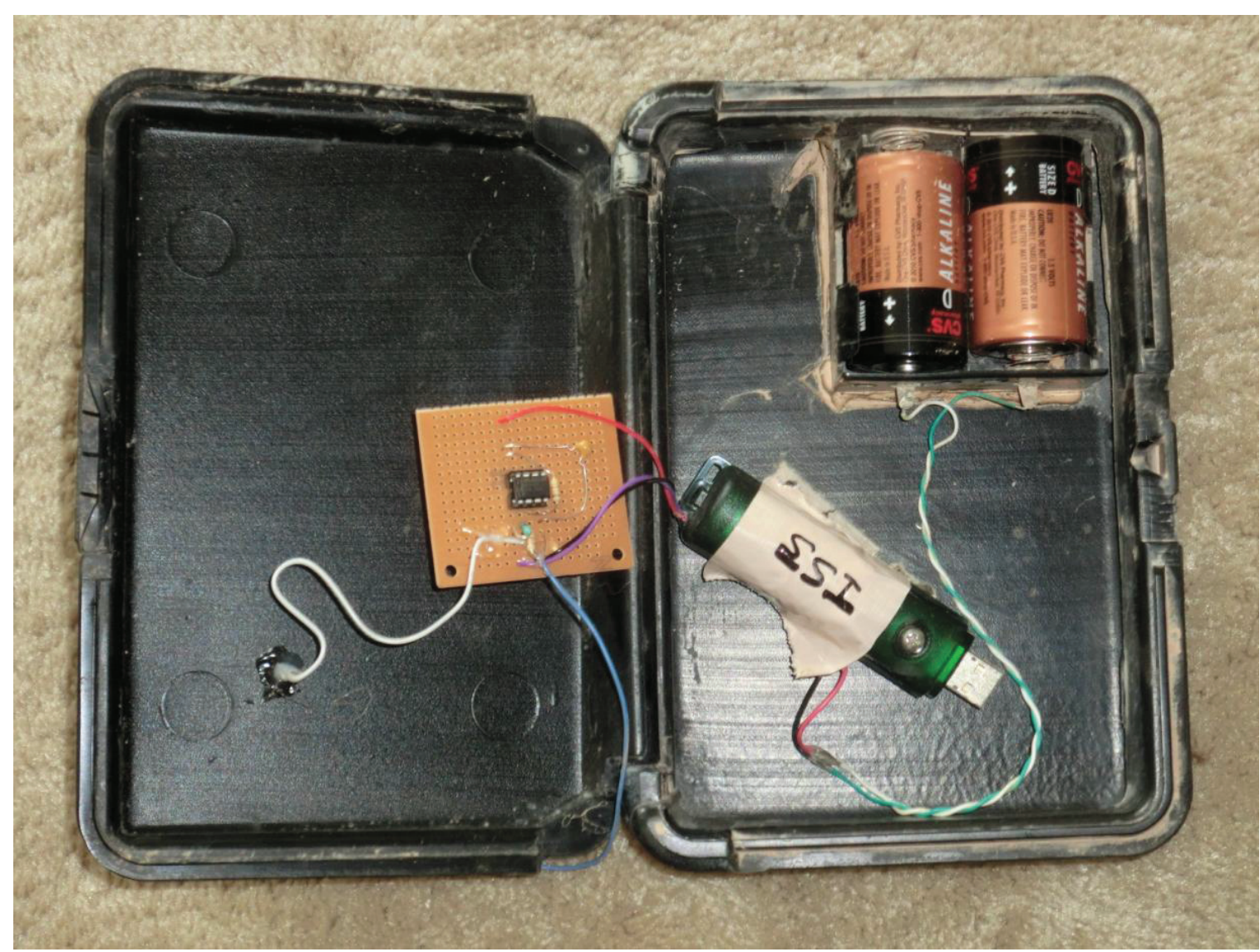

Figure 3. The instrumentation package comprises a datalogger (a large USB datastick) at lower right, powered by two alkaline D-cells at upper right. An analog logging channel is wired to the point discharge current sensor on the circuit board at left, which is grounded to a wire mesh (not shown). The white wire at left is the input discharge wire. 
100 For four loggers, this measurement channel was wired to a point discharge current sensor, using a

101 simple copper wire as a current collection antenna. Low voltage operation was required, since the

102 logger provided only 3.3V regulated power, and low current consumption was desired to permit long

103 duration operation. Further, low input bias current circuitry was required for this measurement: we

104 therefore selected the MAXIM MAX-407 electrometer-specification operational amplifier (<0.1 pA bias).

105 For this application, an aluminium mesh was attached to the case for grounding and screening, and to

106 limit surface charging (figure 3). The current-collecting electrode was a simple PVC-insulated tinned

107 copper wire projected vertically, further isolated from the case by a Teflon sleeve. About $7 \mathrm{~mm}$ of tinned

108 conductor was exposed, $7 \mathrm{~cm}$ above the upper surface of the case and thus $10 \mathrm{~cm}$ above the ground

109 (figure 4). The unit was simply placed on the soil. 


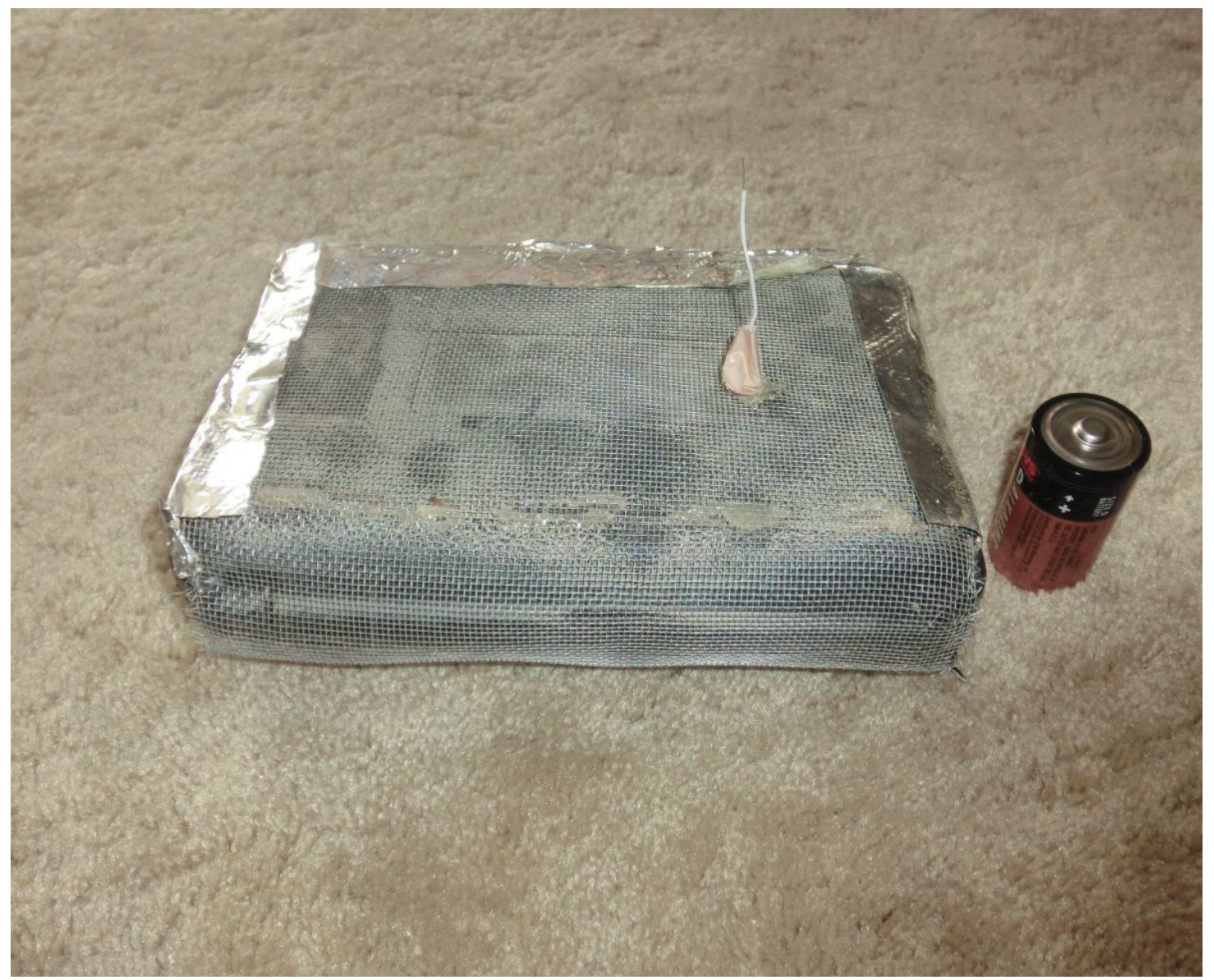

Figure 4. The logger as it was deployed in the field. The sensing wire projects $\sim 7 \mathrm{~cm}$ up from the wire mesh through an insulating Teflon sleeve (here hidden by some stiffening tape)

Because there may be a wide dynamic range of currents to measure, (e.g. Marlton et al., 2013 show point discharge currents from fA up to $\mu \mathrm{A}$, depending on electrified cloud activity) two units (I51, I54) were configured (figure 5) as a logarithmic current amplifier inspired by the point discharge sensor design of Marlton et al. (2013). We used a pair of back-to-back near-infrared LEDs as the feedback element in this instance. Although previous applications favoured green LEDs as having a stronger response (Marlton et al., 2013; Acharaya and Aggarwal, 1996), their forward voltage drop was too high for the present low-voltage operation, and so 940nm near-IR LEDs, with a smaller bandgap ( 1.3V) were needed. Although 'air-wiring' the electrode to the op-amp is necessary for ultra-low current 
measurements, this is much less necessary for the typical currents found in atmospheric point discharge (Marlton et al, 2013) and mechanical robustness for field deployment becomes the paramount consideration; the electrode wire was therefore simply soldered to the circuit board conventionally. For simplicity the temperature compensation circuits of Marlton et al. (2013) were not implemented.

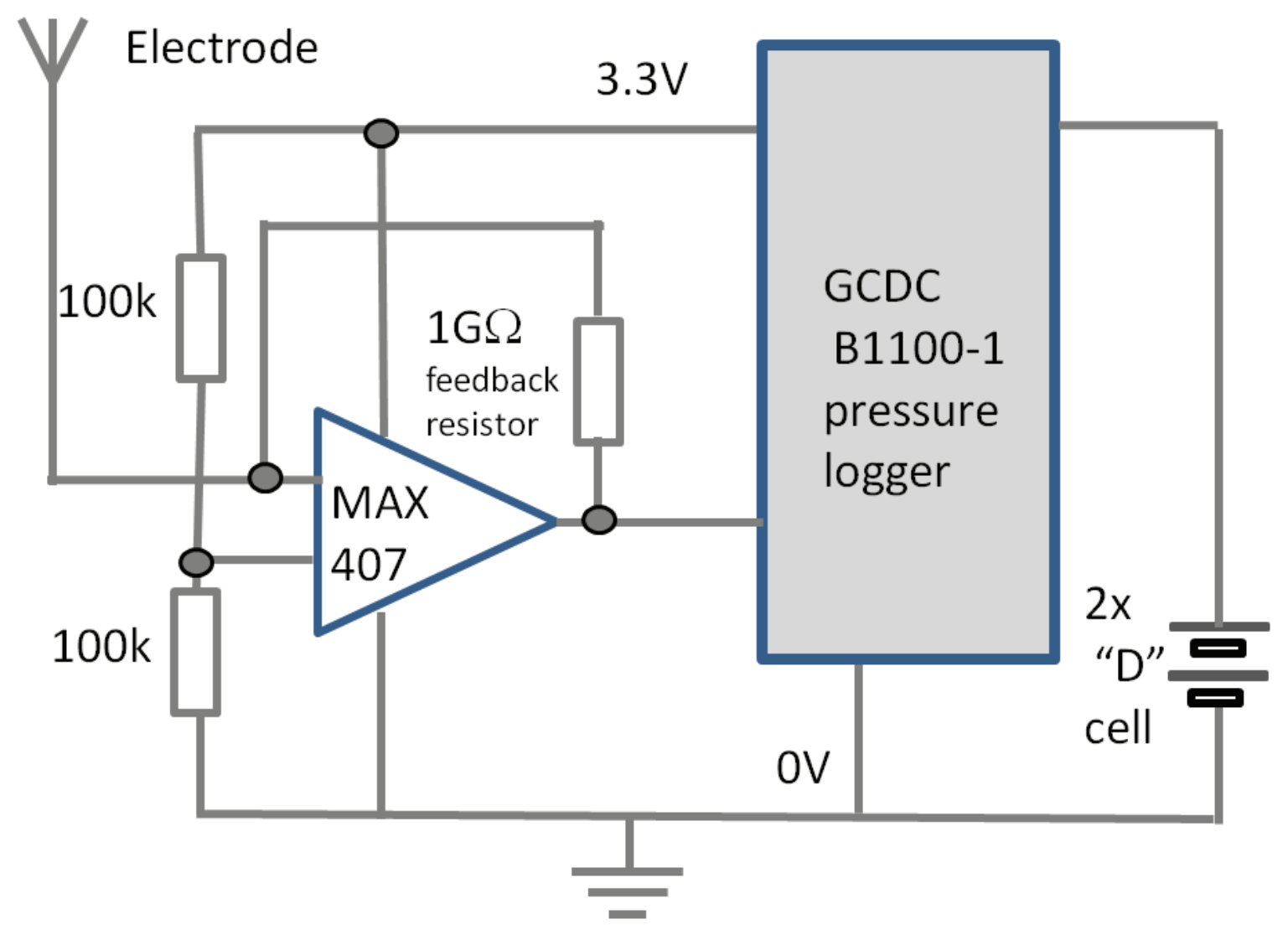

Figure 5. Schematic diagram of the point discharge sensor current amplifier and pressure sensor logger setup. In this instance, the feedback component is a $1 \mathrm{G} \Omega$ resistor. In two other units this was replaced by a pair of LEDs. A $100 \mathrm{nF}$ decoupling capacitor (which is connected between across the op-amp supply rails) is not shown.

In addition, two units were configured as simple current amplifiers, one (a low sensitivity device) with a $10 \mathrm{M} \Omega$ feedback resistor (giving a 150nA full-scale reading for the $\sim 1.5 \mathrm{~V}$ voltage swing permitted by the sensor power supply) and another (a high sensitivity device) with a 1 G $\Omega$ resistor, giving a 1500 pA full- 
136

137

scale reading and a $1 \mathrm{pA}$ resolution. All the loggers were deployed at the beginning of May, the start of the main dust devil season, and retrieved in mid-June, with the intent of avoiding monsoon rains.

It was found upon recovery that one of the LED-based sensors had become waterlogged, apparently by a rain event in early May, and no useable data were retrieved. The low sensitivity device operated as intended, but had inadequate sensitivity to detect electrical signatures associated with dust devils. Although one wide-range LED unit did record a vortex encounter with a strong electrical signal (figure 6), the data from this unit was generally rather noisy and few coherent vortices were detected.
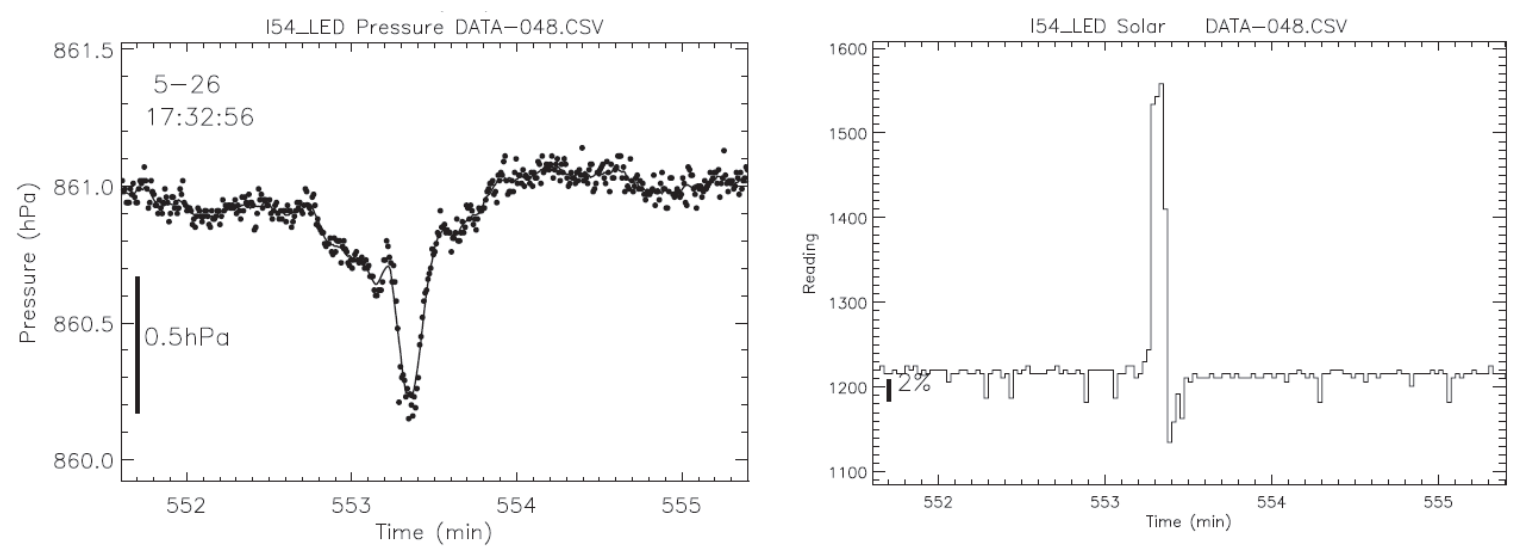

Figure 6. Dust devil encounter recorded by one of the LED-equipped units. (Left) pressure history over 4 minutes during the afternoon of $26^{\text {th }}$ May 2016, with a sharp dip of $\sim 0.7 \mathrm{hPa}$ (0.7 mbar) corresponding to a close passage of a dust devil vortex (the larger irregular dip may be due to a cycloidal migration path giving a complex distance history - e.g. Lorenz, 2013). (Right) a large electrical disturbance is seen coincident with the close passage of the devil : from the I-V characteristics of near-IR diodes (Acharaya and Aggarwal 1996) the $\sim 320 \mathrm{mV}$ signal is estimated to correspond to a current of the order of $1 \mathrm{nA}$.

The high sensitivity linear unit (in fact, employing the rather simple circuit of figure 6) appears to have functioned as intended, and recorded many dust devil encounters with clear electrical signatures. Results from this device are discussed in the remainder of this paper. 
3. Results

159 The pressure record was used to identify close vortex passages, following the methodology of Lorenz

160 and Jackson (2015), namely through a 6-s average reading being lower than the mean of the 30s before

161 and after by 0.2 mbar. This algorithm fails to detect very brief pressure dips (due to very small and/or

162 rapidly-advected vortices) and very long duration events, but maintains a low rate of false detections.

It is seen (figure 7) that a typical electrical signature is a positive voltage excursion of a couple of seconds (corresponding to a negative current, since the amplifier is in an inverting configuration), followed by an instantaneous swing to negative and then recovery back to zero. This is consistent with the base of dust devils having a generally negative space charge. Thus as the dust devil approaches, a positive current is induced, switching to a negative current as the dust devil makes closest approach and recedes. Only in a few instances (e.g. figure 8 ) was this pattern not seen, possibly because the event was so brief that the 1-s sampling interval failed to capture the approach phase.

171
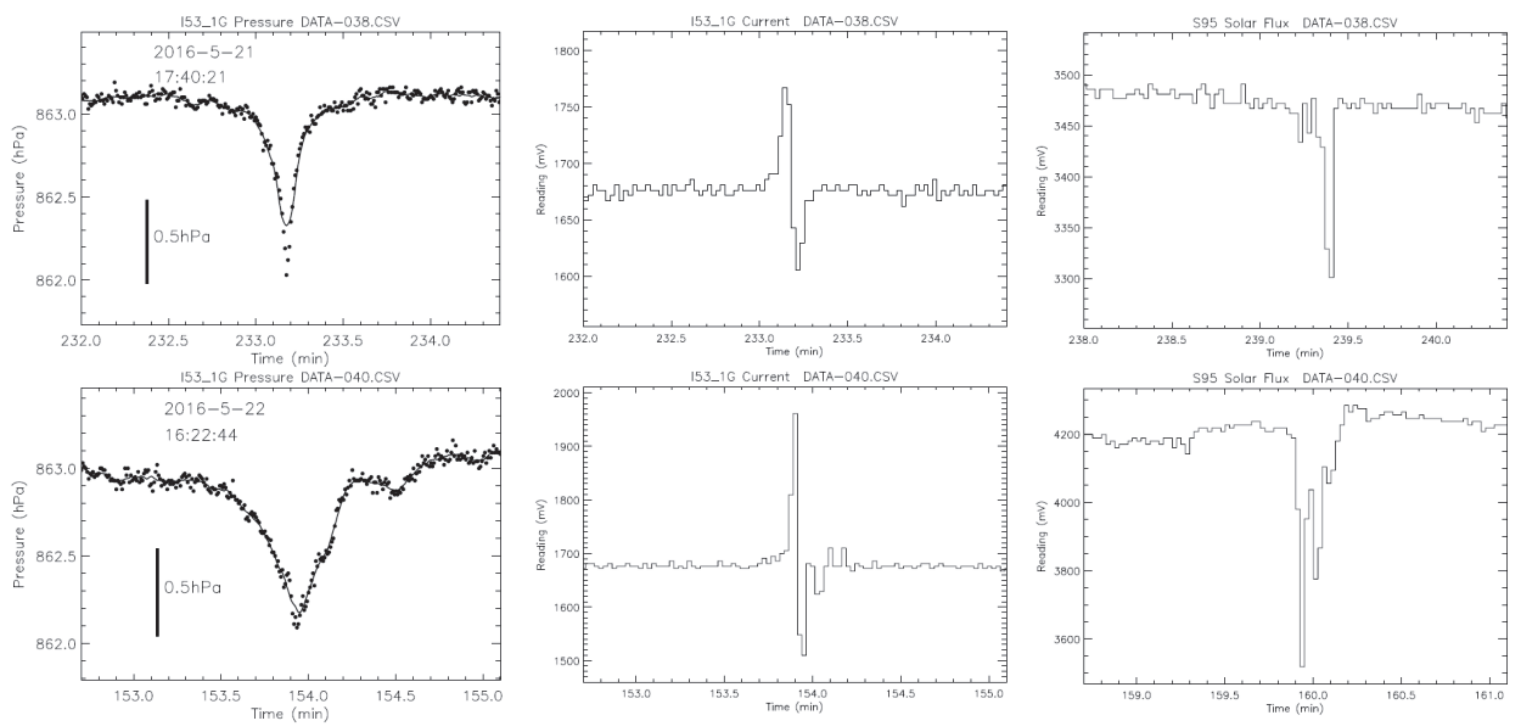

172 
173 Figure 7. Two example encounters on 21 and 22 May respectively. The columns are (left to right) the

174 pressure recorded at the high sensitivity station, the voltage on the current monitor (the values

175 correspond to the current in pA (offset by $50 \%$ of the supply voltage, or 1670 mV), and the short circuit

176 current of a solar cell on a nearby logger, illustrating the obscuration of sunlight by the dust plume

177 associated with the vortex (note that the periods shown are the same for each set of three plots : the

178 timebase of the solar cell is offset by 6 minutes : synchronization was checked by matching the pressure

179 record at the solar cell with that on the current meter). The 'heartbeat' shape of the current curve, and

180 the correlations of this event with the vortex pressure signal and the dust obscuration, are evident. 

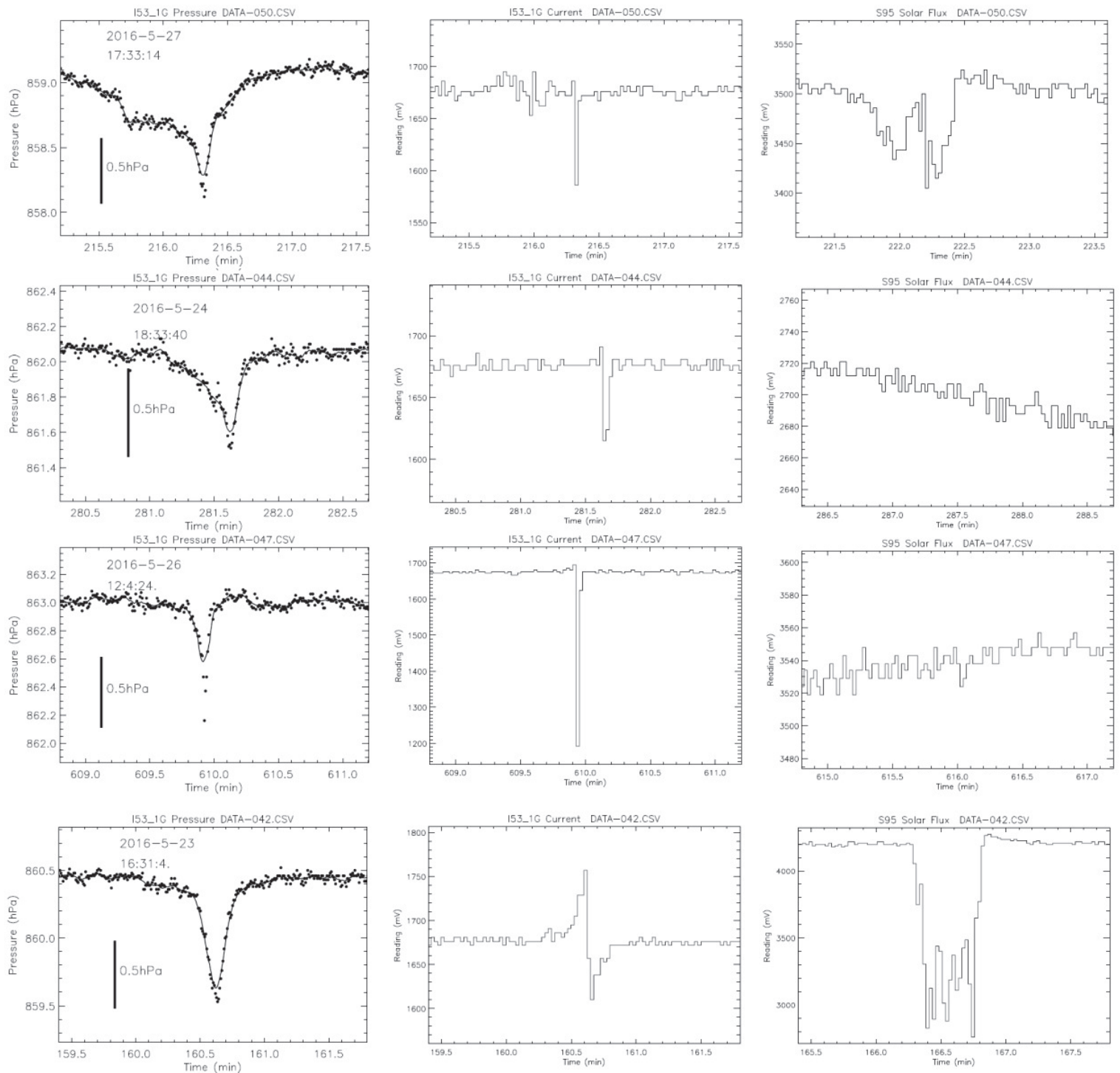

Figure 8. Further example encounters, as figure 7. Top, the same event detected by the wide-range LED unit (figure 6) in this case recorded by the nearby $1 G \Omega$ high sensitivity sensor. Only a brief main current excursion is seen, although with some irregular precursors. The solar record (right, again the difference in numeric timebase is not significant) indicates either multiple encounters with a meandering devil (Lorenz, 2013), or possibly a multi-core vortex or the two walls in a diametric encounter (Lorenz and Jackson, 2015). Lines 2 and 3 show brief, single-sided dips : there was no corresponding dust obscuration event, although this could be due to the non-colocation of the dust measurement. Bottom, again a 'heartbeat' signature, with the solar flux record showing a rather strong (35\%) obscuration lasting some 30 seconds. 
193 This characteristic pattern argues for a space charge in the dust plume being advected across the sensor:

194 although the logger is low on the ground such that the wire could be impacted directly by charged sand,

195 this appears not to be a significant factor in the signatures associated with vortex passage. Occasional

196 single-sample spikes of $+/-20 \mathrm{mV}$ were seen that were not associated with measurable vortex passages,

197 and cannot be excluded as impacts of sand, grass or other debris. However, we note that the noise level

198 on the $1 \mathrm{G} \Omega$ logger current channel was in any case around $10 \mathrm{mV}$ (i.e. $10 \mathrm{pA}$ ) both day and night.

199

200 Ambient weather conditions were recorded at the nearby Jornada SCAN weather station operated by

201 the National Water and Climate Center

202 (http://wcc.sc.egov.usda.gov/nwcc/site?sitenum=2168\&state=nm) and noted alongside the vortex

203 event parameters in table 1. Generally winds were from the south with average speeds of $\sim 10 \mathrm{mph}$

204 ( $\sim 5 \mathrm{~m} / \mathrm{s}$, typical for favorable conditions for dust devil formation) and the humidity was low. 
$\stackrel{ }{\sim}$

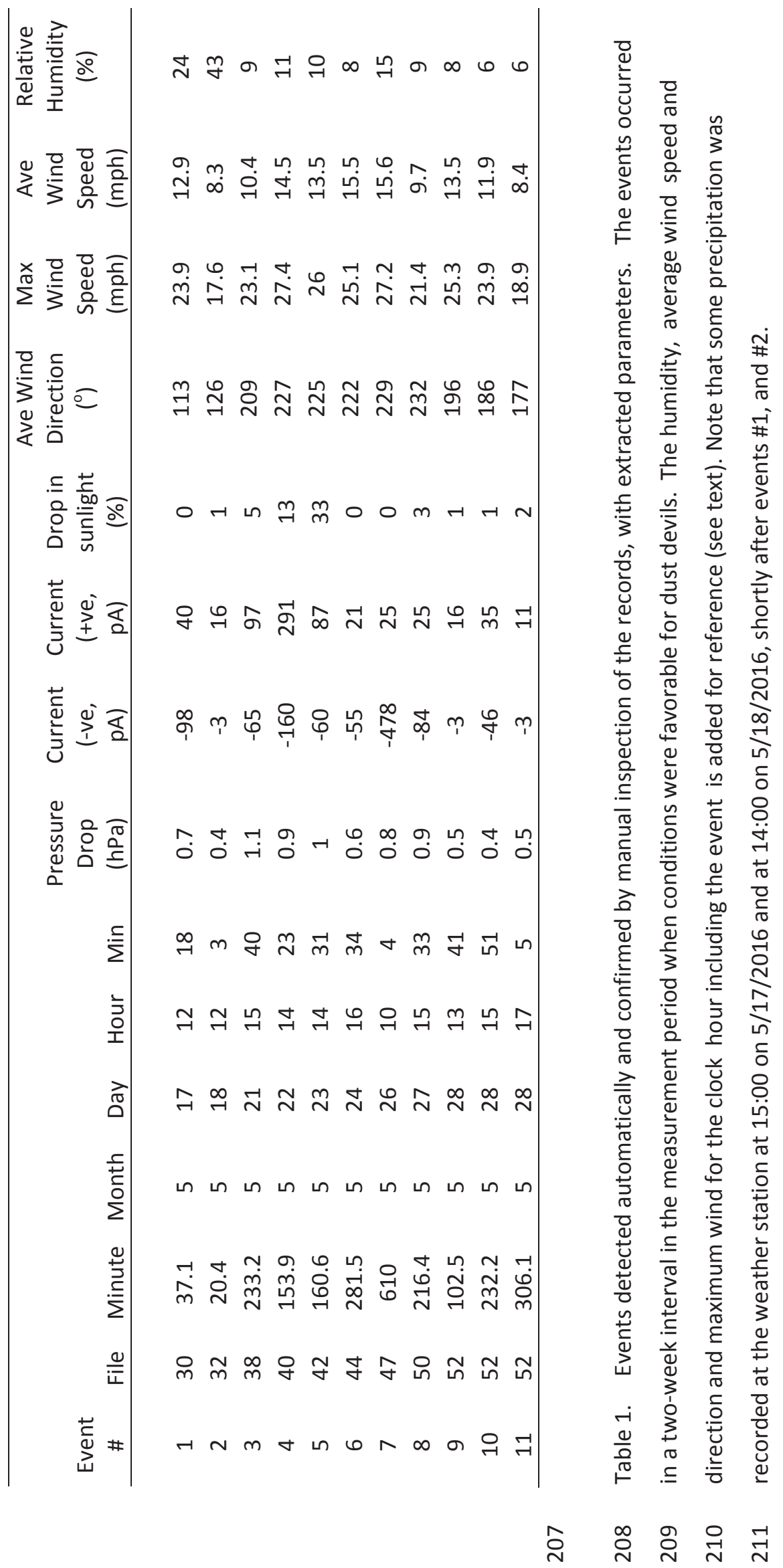


It is seen that the electrical signature is much shorter in duration than the pressure excursion, which varies approximately as $\sim R^{2} /\left(R^{2}+d^{2}\right)$ where $R$ is the dust devil half-diameter and $d$ is the distance from the sensor to the center of the devil. (The dust devil diameter is obvious in well-formed cylindrical vortices as the 'wall' of dust, and corresponds to a pressure drop equal to half that at the vortex center.) This is not surprising, in that the spatial extent of the electrical disturbance would be expected to correspond closely to that of the dust which is typically the half-pressure radius, whereas a detectable pressure excursion typically extends several times further (to roughly where the pressure drop is a tenth of that at the core).

Crozier (1970) note that pulses in observed electric field were observed when the dust loading was temporarily enhanced when the devil encountered areas of enhanced dust availability and Esposito et al. (2016) document a number of dust devil encounters in Morocco, showing that in general the dust loading correlated with electric field perturbation, and the two were negatively correlated with relative humidity. Fields of thousands to tens of thousands of $\mathrm{V} / \mathrm{m}$ were recorded during dust devil encounters. We would expect that in general the discharge current would correlate linearly with the local electric field (e.g. Large and Pierce, 1956; Kirkman and Chalmers, 1957). Wind speed is also a significant factor in influencing the current, although different expressions for this dependence, and the dependence on the height of the point above the ground, have been proposed (e.g. Chalmers and Mapleson, 1955). It should be noted, however, that the correlation of 'fair weather' point discharge current with windspeed merely leads to an increase in the current for a given electrical field, and would not explain the change in polarity (the 'heartbeat') we observe which appears to be associated with dust devil passage specifically. 


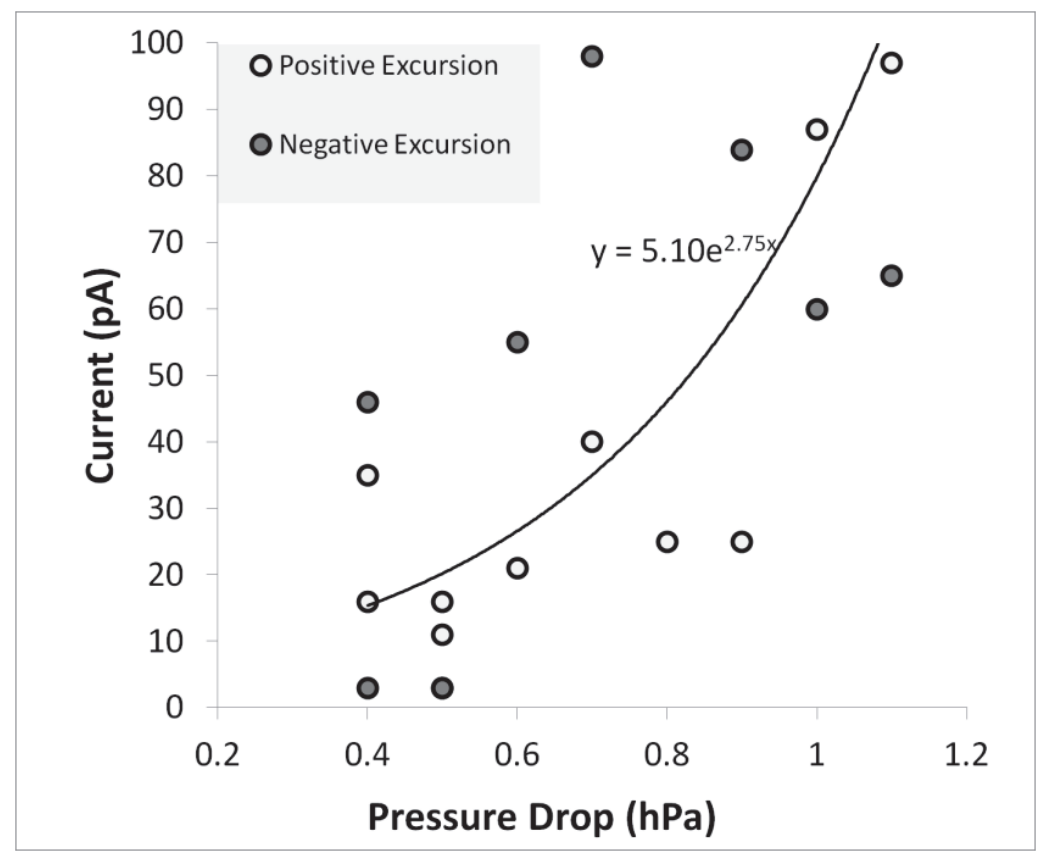

Figure 9. The maximum positive and negative discharge current is shown as a function of the core pressure drop. It is seen that both are correlated, although the data do not permit a robust discrimination of the functional dependence. An example exponential fit is shown for the positive excursion, but a linear dependence on pressure above some threshold value would also be an adequate fit. (NB the outlier, event \#7, is not shown and is excluded from the fit).

Figure 9 shows the correlation of the peak negative current with the peak pressure drop. Note that the pressure drops are slightly larger than typical : a survey at this same site in June 2013 (Lorenz et al., 2015) found that $0.4 \mathrm{mbar}$ encounters occurred at a rate of about 30 per 100 days, while 1 mbar encounters were about ten times less frequent.

We find some correlation (figure 9) between the optical effect of the dust loading (which was not directly determined, since the solar obscuration was measured at a site offset by about $15 \mathrm{~m}$ from the point discharge sensor) and discharge current. We may note that Esposito et al. (2016) documented a somewhat linear correlation of electric field with dust loading for encounters with a fixed relative humidity. 


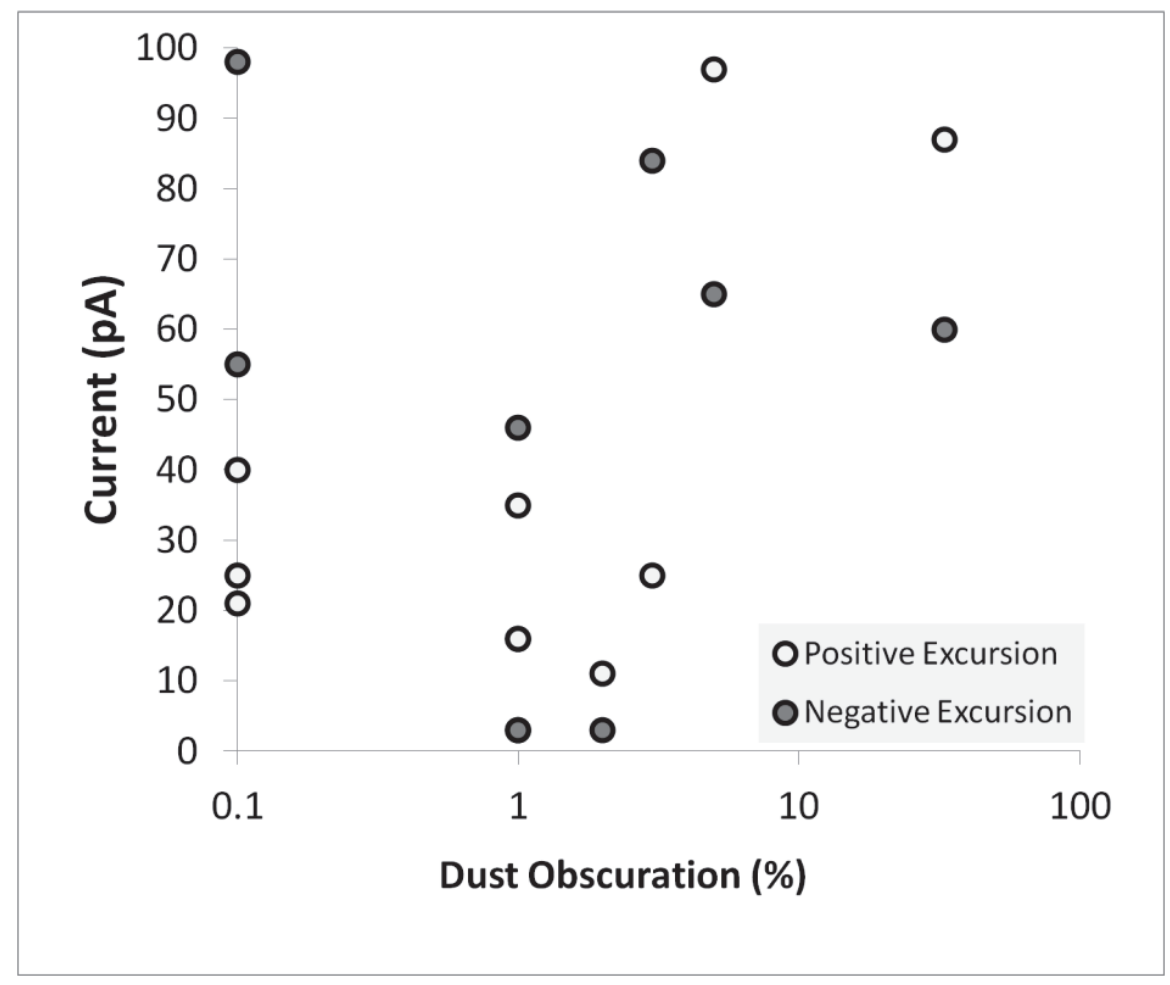

257 Figure 10. Correlation of peak current with dust content measured nearby. The fact that the

258 measurements were not co-located is probably the reason for the set of points with negligible $(0.1 \%$ on

259 this logarithmic plot) dust obscuration. When dust obscuration was detected, it seems positively

260 correlated with the current.

262 The observation period was about 40 days, or $\sim 4$ million seconds, during which 11 events were detected

263 with electrical disturbances of some tens of picoAmps each lasting 10 seconds or so. Thus conditions

264 were disturbed for a fraction of about $3 \times 10^{-5}$ of the time (30 millionths). This is in quite good agreement

265 with the area fraction of about $1 \times 10^{-5}$, occupied by the typical dust devil population on Earth (e.g. Lorenz

266 and Jackson, 2016) described by a -1.6 cumulative power law in diameter between 1 and 100m in

267 diameter. It follows that the time-averaged current from a point is $\sim 10^{-16} \mathrm{~A}$. 
268

269

270

271

272

273

274

275

276

277

278

279

280

281

282

283

284

285

286

287

288

289

290

291

292

293
The introduction of these currents to exposed conductors may have implications for the operation of unattended ground sensors for security applications. If these dust-devil-triggered currents cause spurious detections, a false alarm rate of 0.25/day during dust devil season may be expected.

\section{Conclusions}

Field testing has demonstrated that a very simple and compact sensor circuit can make useful measurements of dust devil electrical properties. The compact and simple electrode and amplifier configuration, presently arranged as a point discharge current meter, could be modified to measure electric fields, or could be made more sensitive to small currents. The configuration shown here gives robust indication of dust, and could be adapted to e.g. operation on an airborne platform such as an Unmanned Aerial Vehicle (UAV), and is inexpensive enough (unlike a conventional field mill) to allow its replication in large numbers, in principle permitting multiple measurements using an array of sensors. An important step in future work would be to compare the current with independent electric field measurements. One might hope in a more extended campaign to be able to resolve the vortex 'wall' where dust density is locally high.

\section{Acknowledgements}

This work was funded by NASA through the Mars Fundamental Research Program grant number NNX12AI04G. We thank Martina Klose for assistance in the field. We thank the Astronomy Department of NMSU for assistance with shipping and the NASA Planetary Data System Atmospheres Node for hosting dust devil data. Additional support was provided by Jornada Basin LTER (supported by National Science Foundation Grant DEB-1235828). KAN acknowledges a NERC Independent Research Fellowship (NE/L011514/1). 


\section{References}

Acharya, Y.B. and Aggarwal, A.K., 1996. Logarithmic current electrometer using light emitting diodes. Measurement Science and Technology, 7(2), 151-156

Atreya, S. K.; A.-S. Wong, N. O. Renno, W. Farrell, G. Delory, D. Sentman, S. Cummer, J. Marshall, S. C. Rafkin, D. C. Catling,; 2006. Oxidant Enhancement in Martian Dust Devils and Storms: Implications for Life and Habitability, Astrobiology, Volume 6, Issue 3, pp. 439-450.

Baddeley, P. F. H. (1860), Whirlwinds and Dust Storms of India, Bell and Daldey, London.

Balme, M. and R. Greeley, 2006. Dust Devils on Earth and Mars, Reviews of Geophysics, 44, RG3003

Barth, E.L., Farrell, W.M. and Rafkin, S.C., 2016. Electric field generation in Martian dust devils. Icarus, 268, pp.253-265.

Chalmers, J. A. and W. W. Mapleson, 1955. Point-discharge currents from a captive balloon, J. Atmospheric Terrestrial Physics, 6, 149=159

Crozier, W.D., 1964. The electric field of a New Mexico dust devil. Journal of Geophysical Research, 69(24), 5427-5429.

Delory, G. T., W.M. Farrell, S.K. Atreya, N.O. Renno, A.-S. Wong, S.A. Cummer, D.O. Sentman, J.B. Marshall, S.C.R. Rafkin, D.C. Catling,2006. Oxidant Enhancement in Martian Dust Devils and Storms: Storm Electric Fields and Electron Dissociative Attachment, Astrobiology, Volume 6, Issue 3, pp. 451-462.

Ellehoj, M. D. , H. P. Gunnlaugsson, P. A. Taylor, H. Kahanpää, K. M. Bean, B. A. Cantor, B. T. Gheynani, L. Drube, D. Fisher, A.-M. Harri, C. Holstein-Rathlou, M. T. Lemmon, M. B. Madsen, M. C. Malin, J. Polkko, P. H. Smith, L. K. Tamppari, W. Weng, and J. Whiteway, Convective vortices and dust devils at the Phoenix Mars mission landing site, Journal of Geophysical Research, 115, EO0E16, 2010 
Esposito, F., Debei, S., Bettanini, C., Molfese, C., Arruego Rodriguez, I., Colombatti, G., Harri, A.M., Montmessin, F., Wilson, C., Aboudan, A. and Abbaki, S., 2014. The DREAMS Experiment of the ExoMars 2016 Mission for the study of Martian environment during the dust storm Season. LPI Contributions, 1791, p.1246.

Esposito, F., Molinaro, R., Popa, C.I., Molfese, C., Cozzolino, F., Marty, L., Taj-Eddine, K., Di Achille, G., Franzese, G., Silvestro, S. and Ori, G.G., 2016. The role of the atmospheric electric field in the dust-lifting process. Geophysical Research Letters. 43, 5501-5508, doi:10.1002/2016GL068463

Farrell, W. M. et al. (2003) "A simple electrodynamic model of a dust devil" Geophysical Research Letters 30, 2050.

Farrell, W. M. et al. (2006) "A model of the ULF magnetic and electric field generated from a dust devil" Journal of Geophysical Research 111, E11004

Harrison, R.G., Barth, E., Esposito, F., Merrison, J., Montmessin, F., Aplin, K.L., Borlina, C., Berthelier, J.J., Déprez, G., Farrell, W.M. and Houghton, I.M.P., 2016. Applications of electrified dust and dust devil electrodynamics to Martian atmospheric electricity. Space Science Reviews, pp.1-47.

Kirkman, J.R. and Chalmers, J.A., 1957. Point discharge from an isolated point. Journal of Atmospheric and Terrestrial Physics, 10(5-6), 258-265.

Kok, J. F. and Renno, N. O. (2009) "Electrification of windblown sand on Mars and its implications for atmospheric chemistry" Geophysical Research Letters 36, L05202. 
343

Large, M.I. and Pierce, E.T., 1957. The dependence of point-discharge currents on wind as examined by a new experimental approach. Journal of Atmospheric and Terrestrial Physics, 10(5-6), 251-257.

Lorenz, R. D., 2012. Observing Desert Dust Devils with a Pressure Logger, Geoscientific Instrumentation, Methods and Data Systems, 1, 209-220

Lorenz, R. D., 2013. Irregular Dust Devil Pressure Drops on Earth and Mars: Effect of Cycloidal Tracks, Planetary and Space Science, 76, 96-103, 2013

Lorenz, R. D., 2016. Heuristic Estimation of Dust Devil Vortex Parameters and Trajectories from Single-Station Meteorological Data: Application to InSight at Mars, Icarus, 271, 326-337

Lorenz, R. D. and B. K. Jackson, 2015. Dust Devils and Dustless Vortices on a Desert Playa Observed with Surface Pressure and Solar Flux Logging, GeoResJ, 5, 1-11

Lorenz, R. D. and B. K. Jackson, 2016. Dust Devil Populations and Statistics, Space Science Reviews, in press

Lorenz, R.D., Neakrase, L.D. and Anderson, J.D., 2015. In-situ measurement of dust devil activity at La Jornada Experimental Range, New Mexico, USA. Aeolian Research, 19, 183-194.

Lorenz, R. D., M. R. Balme, Z. Gu, H. Kahanpää, M. Klose, M. Kurgansky, M. R. Patel, D. Reiss, A. P. Rossi, A. Spiga,T. Takemi, W. Wei. 2016. History and Applications of Dust Devil Studies, Space Science Reviews, in press doi:10.1007/s11214-016-0239-2

Marlton, G., R. G. Harrison and K. Nicoll, 2013. Note: Atmospheric point discharge current measurements using a temperature-compensated logarithmic current amplifier, Review of Scientific Instrumentss, 84, 066103 
373 Nicoll, K. A., R. G. Harrison and Z. Ulanowski, Observations of Saharan dust layer

374 Electrification, Environment Research Letters, 6, /014001, 2011. doi:10.1088/1748-

$3759326 / 6 / 1 / 014001$

376

377 Snow, J. T. and T. McClelland, Dust devils at White Sands Missile Range, New Mexico 1.

378 Temporal and Spatial Distributions, 1990. J. Geophysical Research, 95, 13,707-13,721 